\title{
Hermitian Metrics \\ of Negative Holomorphic Sectional Curvature on Some Hyperbolic Manifolds
}

\author{
Chi-Keung Cheung * \\ Department of Mathematics, University of Michigan, Ann Arbor, Michigan 48109, USA
}

\section{Introduction}

It is well-known that if a complex manifold admits a Hermitian metric with holomorphic sectional curvature bounded above by a negative constant, then it is hyperbolic $([6,8,15])$. The converse is still open. Some partial results are known: In [6], Grauert and Reckziegel constructed a Hermitian metric in a neighborhood of a fibre of an analytic family of compact Riemann surfaces of genus $\geqq 2$ over a Riemann surface, such that the metric has negative holomorphic sectional curvature in that neighborhood. Cowen [4] extended the result to $n$ dimensions, but the metric still only exists locally in a neighborhood of a fibre. There has been no global result, except for related works by Deschamps-Martin [5] and Schneider [13], who proved that the Kodaira surfaces of general type [9] have negative tangent bundle in the sense of Grauert. The purpose of this note is to show that for some types of compact hyperbolic manifolds, we can construct a Hermitian metric on them with everywhere negative holomorphic sectional curvature. In particular, Kodaira's surfaces of general type [9] and the example of Grauert and Reckziegel (mentioned above) are included as special cases. Still with Kodaira surfaces, we will show in the last section that they can in fact be given a Kähler metric with negative holomorphic sectional curvature. In contrast with the proof of Theorem 1, the proof of the latter fact is by an explicit construction.

This work is part of the author's Ph.D. thesis written under the supervision of Prof. $\mathrm{H}$. Wu, to whom the author would like to express the deepest gratitude for all his help, support, encouragement and instruction.

\section{Definitions and Statement of Results}

Let $\omega$ be a Hermitian metric on an $n$-dimensional complex manifold with $\omega$ $=\sum g_{i j} d z^{i} d \bar{z}^{j}$ in local coordinates, then the coefficients of the curvature tensor are given by:

$$
\mathbf{R}_{i \bar{j} k \bar{l}}=-\frac{\partial^{2} g_{i \bar{j}}}{\partial z^{k} \partial \bar{z}^{l}}+\sum g^{q \bar{p}} \frac{\partial g_{i \overline{\bar{p}}}}{\partial z^{k}} \frac{\partial g_{\bar{j} \bar{j}}}{\partial \bar{z}^{l}} .
$$

\footnotetext{
* Research partially supported by National Science Foundation
} 
The holomorphic sectional curvature at a point $\mathbf{p}$ in the direction $\left(\zeta^{1}, \zeta^{2}, \ldots, \zeta^{n}\right)$ is given by:

$$
\frac{\sum_{i, j, k, l=1}^{n} \mathbf{R}_{i \bar{j} k \bar{l}}(\mathbf{p}) \zeta^{i} \overline{\zeta^{j}} \zeta^{k} \bar{\zeta}}{\sum_{i, j, k, l=1}^{n} g_{i \bar{j}}(\mathbf{p}) g_{k \bar{l}}(\mathbf{p}) \zeta^{i} \overline{\zeta^{j}} \zeta^{k} \bar{\zeta}} .
$$

Theorem 1. Let $\pi: \mathbf{X} \rightarrow \mathbf{Y}$ be a holomorphic map of a compact complex manifold $\mathbf{X}$ into a complex manifold $\mathbf{Y}$ which has a Hermitian metric of negative holomorphic sectional curvature. Assume also that $\pi$ is of maximal rank everywhere and there exists a smooth family of Hermitian metrics on the fibres, which all have negative holomorphic sectional curvature. Then there exists a Hermitian metric on $\mathbf{X}$ with negative holomorphic sectional curvature everywhere.

We note that the assumption of the theorem immediately imply that $\mathbf{X}$ is a hyperbolic manifold. In greater detail, since $\pi$ is of maximal rank and $\mathbf{X}$ is compact, it is well-known that $\pi$ must be onto and $\mathbf{Y}$ must be compact. Hence $\mathbf{Y}$ having a Hermitian metric of negative holomorphic sectional curvature, implies that $\mathbf{Y}$ is hyperbolic. Since each fibre $\mathbf{X}_{t} \equiv \pi^{-1}(t)(t \in \mathbf{Y})$ is compact and since each $\mathbf{X}_{t}$ is also assumed to have a Hermitian metric of negative holomorphic sectional curvature, each $\mathbf{X}_{t}$ is hyperbolic. Thus it follows that $\mathbf{X}$ is hyperbolic (Use Brody's Theorem [2], for example). For a later reference, we should also explain the precise meaning of the existence of a smooth family of Hermitian metrics on the fibres. For each $\mathbf{p} \in \mathbf{X}$, we can choose a neighborhood $\mathscr{U}$ of $\pi(\mathbf{p})$ so that if $\left\{t^{1}, \ldots, t^{m}\right\}$ are complex coordinates on $\mathscr{U}$, then on $\pi^{-1}(\mathscr{U})$ we have complex coordinates $\left\{t^{1} \circ \pi, \ldots, t^{m} \circ \pi, z^{1}, \ldots, z^{s}\right\}$ so that the restrictions of $\left\{z^{1}, \ldots, z^{s}\right\}$ to $\mathbf{X}_{t} \cap \pi^{-1}(\mathscr{U})$ for each $t$ give local coordinates on the latter. By a common abuse of notation, we shall write $t^{i}$ in place of $t^{i} \circ \pi$. Now let $g_{t}$ be a Hermitian metric on $\mathbf{X}_{t}$ for each $t \in \mathbf{Y}$. We say $\left\{g_{t}\right\}$ is a smooth family if relative to the coordinates $\left\{t^{i}, z^{\alpha}\right\}$ on each $\pi^{-1}(\mathscr{U})$ as above, there exist $\mathscr{C}^{\infty}$ functions $g_{\alpha \bar{\beta}}(z, t)$ for $\alpha, \beta=1, \ldots, s, z=\left(z^{1}, \ldots, z^{s}\right)$ and $t=\left(t^{1}, \ldots, t^{m}\right)$, so that for each $t$,

$$
g_{t}=\sum_{\alpha, \beta} g_{\alpha \bar{\beta}}(z, t) d z^{\alpha} d \bar{z}^{\beta}\left(\bmod \left\{d t^{i}\right\}\right) .
$$

This definition is easily seen to be equivalent to the usual one as used by Kodaira-Spencer [10].

The conclusion of Theorem 1 is optimal in the sense that if $\pi: \mathbf{X} \rightarrow \mathbf{Y}$ is a holomorphic fibre bundle, then there are no Hermitian metrics on such an $\mathbf{X}$ with negative bisectional curvature. For the proof of this assertion, it suffices to adapt the argument of Yang in [17] to our present situation. More precisely, if $v(y)$ denotes the volume of $\pi^{-1}(y), y \in \mathbf{Y}$, then at the maximum point of $v$ in $\mathbf{Y}$ (using the compactness of $\mathbf{Y}$ here), the argument on p. 132 of [17] would go through unchanged without Yang's Kähler assumption.

For the first corollary, recall that if a compact complex manifold $\mathbf{M}$ has negative first Chern class, the theorem of Aubin and Yau says that $\mathbf{M}$ has a unique Einstein-Kähler metric of Ricci curvature - 1. We call this the canonical Einstein-Kähler metric of $\mathbf{M}$. 
Corollary 1. Let $\pi: \mathbf{X} \rightarrow \mathbf{Y}$ be a holomorphic map of a compact complex manifold $\mathbf{X}$ into a complex manifold $\mathbf{Y}$ which has a Hermitian metric of negative holomorphic sectional curvature. Assume also that $\pi$ is of maximal rank everywhere that each fiber has negative first Chern class and its canonical Kähler-Einstein metric has negative holomorphic sectional curvature. Then there exists a Hermitian metric on $\mathbf{X}$ with negative holomorphic sectional curvature everywhere.

The curvature assumptions on the fibres are not unnatural. They are satisfied for example, if each fibre is a Riemann surface with genus $\geqq 2$, or less trivially, if each fibre is a Kähler manifold of the homotopy type of a compact quotient of an irreducible Hermitian symmetric space of noncompact type of complex dimension $\geqq 2$. The latter uses the strong rigidity theorem of Siu [14]. In fact, the fibres can even be any suitable compact quotient of a Hermitian symmetric space of noncompact type; this has to use the extension of Siu's theorem by Mok [12] and Jost-Yau [7]. In the case of co-dimension one fibres, this Corollary simplifies to:

Corollary 2. Let $\pi: \mathbf{X}^{n} \rightarrow \mathbf{Y}^{n-1}$ be a holomorphic map of a compact complex manifold $\mathbf{X}$ into a complex manifold $\mathbf{Y}$ which has a Hermitian metric of negative holomorphic sectional curvature. Assume also that $\pi$ is of maximal rank everywhere and $\mathbf{X}$ is hyperbolic, then there exists a Hermitian metric on $\mathbf{X}$ with negative holomorphic sectional curvature everywhere.

In particular, when $n=2$, the example of Grauert and Reckziegel and Kodaira's surfaces of general type satisfy these assumptions. In other words, these well-known hyperbolic surfaces have a Hermitian metric with negative holomorphic sectional curvature.

We will first try to construct a Hermitian metric in $\mathbf{X}$, such that it has negative holomorphic sectional curvature along the fiber direction (Sect. 4), then add to it $\lambda$ times the pull-back of the Hermitian metric of $\mathbf{Y}$. We will then try to show that, when $\lambda$ is large enough, the resulting metric is of negative holomorphic sectional curvature in all directions. This idea comes from a simple fact in matrix theory: Suppose $\mathbf{A}$ is a $n \times n$ symmetric matrix with its first coefficient $a_{11}>0$ and

$$
\mathbf{B}=\left[\begin{array}{ccccc}
0 & 0 & 0 & \ldots & 0 \\
0 & & & & \\
0 & & & & \\
\vdots & & & \mathbf{B}^{\prime} & \\
0 & & & &
\end{array}\right]
$$

where $\mathbf{B}^{\prime}$ is an $(n-1) \times(n-1)$ positive definite matrix, then we know that $\mathbf{A}+\lambda \mathbf{B}$ is also positive if $\lambda$ is large enough. (It suffices to check the determinants of all principal minors, but since $\mathbf{B}^{\prime}$ is positive definite, so we only have to check $\operatorname{det}(\mathbf{A}+\lambda \mathbf{B})=\lambda^{n-1} a_{11} \operatorname{det} \mathbf{B}^{\prime}+\mathbf{O}\left(\lambda^{n-2}\right)$ which is $>0$ if $\lambda$ is large enough.) Note that this argument depends crucially on the fact that the first column and first 
row of $\mathbf{B}$ are zero. In the geometric situation below, we will see that the same phenomenon reappears.

In Corollary 2, when $\mathbf{X}$ is a Kodaira surface [9], we shall make an improvement:

Theorem 2. There exists a Kähler metric with negative holomorphic sectional curvature on the Kodaira surfaces of general type.

\section{Some Lemmas about Negative Holomorphic Sectional Curvature}

Lemma 1. Let $\mathbf{M}$ be a $n$-dimensional Hermitian manifold and $\mathbf{G}$ the Hermitian metric. Let $\left\{\mathbf{R}_{i j k l}\right\}$ be the coefficients of the curvature tensor and $K_{0}, K_{1}, K_{2}$ be positive constants. Suppose that for some natural number $s<n$, such that at a point $\mathbf{p} \in \mathbf{M}$ :

$$
\begin{aligned}
& \sum_{i, j, k, l=1}^{s} \mathbf{R}_{i \overline{j k} k}(\mathbf{p}) \zeta^{i} \overline{\zeta^{j} \zeta^{k}} \bar{\zeta} \leqq-K_{0} \sum_{i, j=1}^{s} \zeta^{i} \overline{\zeta^{i}} \zeta^{j} \overline{\zeta^{j}} \\
& \quad \text { for all } \zeta^{i} \in \mathbb{C} ., \quad i=1, \ldots, s .
\end{aligned}
$$

(ii) $\quad\left|\mathbf{R}_{i j k l}(\mathbf{p})\right|<K_{1} \quad$ whenever $\min (i, j, k, l) \leqq s$.

$$
\begin{aligned}
& \sum_{\alpha, \beta, \gamma, \delta=s+1}^{n} \mathbf{R}_{\alpha \bar{\beta} \gamma \bar{\delta}}(\mathbf{p}) \zeta^{\alpha} \bar{\zeta}^{\beta} \zeta^{\gamma} \bar{\zeta}^{\delta} \leqq-K_{2} \sum_{\alpha, \beta=s+1}^{n} \zeta^{\alpha} \overline{\zeta^{\alpha}} \zeta^{\beta} \bar{\zeta}^{\beta} \\
& \quad \text { for any } \zeta^{\alpha} \in \mathbb{C} ., \quad \alpha=1, \ldots, n .
\end{aligned}
$$

Then there exists a positive constant $\mathscr{K}$, depending only on $K_{0} / K_{1}$, such that, if $K_{2} / K_{1} \geqq \mathscr{K}$ then $\mathbf{G}$ has neg. hol. sect. (negative holomorphic sectional) curvature at the point $\mathbf{p .}$

Proof. Since we are only interested in the sign of the holomorphic sectional curvature, it suffices to check the numerator of (2). Using assumptions (i) and (ii), we have, $\forall \zeta^{i}, \zeta^{j}, \zeta^{k}, \zeta^{l} \in \mathbb{C}$,

$$
\text { (3) } \begin{aligned}
\sum_{i, j, k, l=1}^{n} & \mathbf{R}_{i \bar{j} k \bar{l}}(\mathbf{p}) \zeta^{i} \overline{\zeta^{j}} \zeta^{k} \bar{\zeta} \leqq-K_{0} \sum_{i, j=1}^{s} \zeta^{i} \overline{\zeta^{i}} \zeta^{j} \overline{\zeta^{j}} \\
& +4 K_{1} \sum_{\alpha, \beta, \gamma=s+1}^{n} \sum_{i+1}^{s}\left|\zeta^{i}\right|\left|\zeta^{\alpha}\right|\left|\zeta^{\beta}\right|\left|\zeta^{j}\right|+6 K_{1} \sum_{\alpha, \beta=s+1}^{n} \sum_{i, j=1}^{s}\left|\zeta^{i}\right|\left|\zeta^{j}\right|\left|\zeta^{\alpha}\right|=\left|\zeta^{\beta}\right| \\
& +4 K_{1} \sum_{\alpha=s+1}^{n} \sum_{i, j, k=1}^{s}\left|\zeta^{i}\right|\left|\zeta^{j}\right|\left|\zeta^{k}\right|\left|\zeta^{\alpha}\right|+\sum_{\alpha, \beta, \gamma, \delta=s+1}^{n} \mathbf{R}_{\alpha \bar{\beta} \gamma \bar{\delta}}(\mathbf{p}) \zeta^{\alpha} \overline{\zeta^{\beta}} \zeta^{\gamma} \bar{\zeta}^{\delta}
\end{aligned}
$$

where the coefficients $4,6,4$ come from the summing of indices. But for any positive numbers $a, b, c, d$, we have:

$$
\begin{aligned}
\left|\zeta^{i}\right|\left|\zeta^{\alpha}\right|\left|\zeta^{\beta}\right|\left|\zeta^{\gamma}\right| & \leqq\left(\left|\zeta^{i}\right|\left|\zeta^{\alpha}\right|\right)^{2}+\left(\left|\zeta^{\beta}\right|\left|\zeta^{\gamma}\right|\right)^{2} \\
& \leqq a^{2}\left|\zeta^{i}\right|^{2}\left|\zeta^{i}\right|^{2}+\frac{1}{a^{2}}\left|\zeta^{\alpha}\right|^{2}\left|\zeta^{\alpha}\right|^{2}+\left|\zeta^{\beta}\right|^{2}\left|\zeta^{\gamma}\right|^{2}
\end{aligned}
$$




$$
\left|\zeta^{i}\right|\left|\zeta^{j}\right|\left|\zeta^{\alpha}\right|\left|\zeta^{\beta}\right| \leqq b^{2}\left|\zeta^{i}\right|^{2}\left|\zeta^{j}\right|^{2}+\frac{1}{b^{2}}\left|\zeta^{\alpha}\right|^{2}\left|\zeta^{\beta}\right|^{2}
$$

$$
\left|\zeta^{i}\right|\left|\zeta^{j}\right|\left|\zeta^{k}\right|\left|\zeta^{\alpha}\right| \leqq c^{2}\left|\zeta^{i}\right|^{2}\left|\zeta^{j}\right|^{2}+\frac{d^{2}}{c^{2}}\left|\zeta^{k}\right|^{4}+\frac{1}{c^{2} d^{2}}\left|\zeta^{\alpha}\right|^{2}\left|\zeta^{\alpha}\right|^{2}
$$

Now choose $a, b, c, d$, so small that:

$$
4(n-s)^{3} a^{2}+6(n-s)^{2} b^{2}+4(n-s) s c^{2}+4 s^{2}(n-s) \frac{d^{2}}{c^{2}} \leqq \frac{1}{2} \frac{K_{0}}{K_{1}} .
$$

Substituting inequalities (4), (5), (6), (7) into (3), we have $\forall \zeta^{i}, \zeta^{j}, \zeta^{k}, \zeta^{l} \in \mathbb{C}$ :

$$
\begin{aligned}
\sum_{i, j, k, l=1}^{n} & \mathbf{R}_{i \bar{j} k \bar{l}}(\mathbf{p}) \zeta^{i} \overline{\zeta^{j}} \zeta^{k} \zeta^{l} \\
\leqq & -\frac{K_{0}}{2} \sum_{i, j=1}^{s} \zeta^{i} \overline{\zeta^{i}} \zeta^{j} \zeta^{j}+\left[\frac{4 K_{1} s(n-s)^{2}}{a^{2}} \sum_{\alpha=s+1}^{n}\left|\zeta^{\alpha}\right|^{4}\right. \\
& +4 K_{1}(n-s) s \sum_{\beta, \gamma=s+1}^{n}\left|\zeta^{\beta}\right|^{2}\left|\zeta^{\gamma}\right|^{2}+\frac{6 K_{1}}{b^{2}} s^{2} \sum_{\alpha, \beta=s+1}^{n}\left|\zeta^{\alpha}\right|^{2}\left|\zeta^{\beta}\right|^{2} \\
& \left.+\frac{4 K_{1}}{c^{2} d^{2}} s^{3} \sum_{\alpha=s+1}^{n}\left|\zeta^{\alpha}\right|^{4}+\sum_{\alpha, \beta, \gamma, \delta=s+1}^{n} \mathbf{R}_{\alpha \bar{\beta} \gamma \bar{\delta}}(\mathbf{p}) \zeta^{\alpha} \overline{\zeta^{\beta}} \zeta^{\gamma} \zeta^{\delta}\right] .
\end{aligned}
$$

Using assumption (iii), it is clear that Lemma 1 follows, if we choose:

$$
\mathscr{K}=2\left(\frac{4 s(n-s)^{2}}{a^{2}}+4(n-s) s+\frac{6 s^{2}}{b^{2}}+\frac{4 s^{3}}{c^{2} d^{2}}\right) .
$$

Lemma 2 (Wu [16], see also [6]). Let $\mathbf{M}$ be a $n$-dimensional complex manifold and let $\mathbf{G}, \mathbf{H}$ be two Hermitian metrics on $\mathbf{M}$ whose holomorphic sectional curvatures $\boldsymbol{K}(\mathbf{G})$ and $\boldsymbol{K}(\mathbf{H})$ satisfy $\boldsymbol{K}(\mathbf{G}) \leqq-L_{1}<0$ and $\boldsymbol{K}(\mathbf{H}) \leqq-L_{2}<0$, where $L_{1}$ and $L_{2}$ are positive constants. Then $\boldsymbol{K}(\mathbf{G}+\mathbf{H}) \leqq-L_{1} L_{2} /\left(L_{1}+L_{1}\right)$.

Also we need a simple result in linear algebra:

Lemma 3. Let $\mathbf{A}$, $\mathbf{B}$ be two $n \times n$ Hermitian matrices, with $\mathbf{A}$ positive definite and $\mathbf{B}$ positive semi-definite. Then, $\forall v \in \mathbb{C}^{n}$, with $v^{*}$ as its transpose conjugate, $v\left(\mathbf{A}+\lambda_{1} \mathbf{B}\right)^{-1} v^{*} \leqq v\left(\mathbf{A}+\lambda_{2} \mathbf{B}\right)^{-1} v^{*}$, if $\lambda_{1} \geqq \lambda_{2}$.

Lemma 4. Let $\mathbf{N}$ be a complex manifold, $\mathbf{H}$ and $\tilde{\mathbf{H}}$ be Hermitian metrics defined on $\mathbf{N}$. Suppose that the metric $\tilde{\mathbf{H}}$ has neg. hol. sect. curvature at the point $\mathbf{p}$. Then if $\lambda$ is large enough, $\mathbf{H}+\lambda \tilde{\mathbf{H}}$ also has neg. hol. sect. curvature at the point p.

Proof. We use the notation of Lemma 2. Let $t$ be a unit vector at $\mathbf{p}$ relative to $\mathbf{H}+\lambda \tilde{\mathbf{H}}$. Let $K(\mathbf{H}+\lambda \mathbf{H}, t)$ denote the holomorphic sectional curvature of $\mathbf{H}+\lambda \tilde{\mathbf{H}}$ in the direction $t$. We shall choose $\lambda$ so large (and independent of $t$ ), so that $\boldsymbol{K}(\mathbf{H}+\lambda \overline{\mathbf{H}}, t)<0$. By Lemma 4 of [16], there exists a 1 -dimensional 
imbedded complex submanifold $\mathbf{N}^{\prime}$ tangent to $t$, such that if $\mathbf{H}^{\prime}$ and $\tilde{\mathbf{H}}^{\prime}$ are the induced metrics of $\mathbf{H}$ and $\tilde{\mathbf{H}}$ on $\mathbf{N}^{\prime}$ respectively (so that $\mathbf{H}^{\prime}+\lambda \tilde{\mathbf{H}}^{\prime}$ is the induced metric of $\mathbf{H}+\lambda \tilde{\mathbf{H}}$ on $\mathbf{N}^{\prime}$ ), then

$$
\boldsymbol{K}(\mathbf{H}+\lambda \tilde{\mathbf{H}}, t)=\boldsymbol{K}\left(\mathbf{H}^{\prime}+\lambda \tilde{\mathbf{H}}^{\prime}\right)(\mathbf{p}) .
$$

Let $z$ be an arbitrary co-ordinate function of $\mathbf{N}^{\prime}$ near $\mathbf{p}$, and let

$$
\mathbf{H}^{\prime}=h d z d \bar{z}, \quad \tilde{\mathbf{H}}^{\prime}=\tilde{h} d z d \bar{z} .
$$

We use the identity of [6] (see also p. 1105 of [16]), namely that

$$
\begin{aligned}
& (\lambda \tilde{h}) h(\lambda \tilde{h}+h)\left(h^{2} \boldsymbol{K}\left(\mathbf{H}^{\prime}\right)+(\lambda \tilde{h})^{2} \boldsymbol{K}\left(\lambda \tilde{\mathbf{H}}^{\prime}\right)-(\lambda \tilde{h}+h)^{2} \boldsymbol{K}\left(\mathbf{H}^{\prime}+\lambda \tilde{\mathbf{H}}^{\prime}\right)\right) \\
& \quad=2\left|h \frac{\partial h}{\partial z}-\lambda \tilde{h} \frac{\partial(\lambda \tilde{h})}{\partial z}\right| \geqq 0
\end{aligned}
$$

to conclude that

$$
\boldsymbol{K}\left(\mathbf{H}^{\prime}+\lambda \tilde{\mathbf{H}}^{\prime}\right)(\mathbf{p}) \leqq\left[\frac{h^{2} \boldsymbol{K}\left(\mathbf{H}^{\prime}\right)+(\lambda \tilde{h})^{2} \boldsymbol{K}\left(\lambda \tilde{\mathbf{H}}^{\prime}\right)}{(h+\lambda \tilde{h})^{2}}\right](\mathbf{p}) .
$$

But since $\boldsymbol{K}\left(\lambda \tilde{\mathbf{H}}^{\prime}\right)=\frac{1}{\lambda} \boldsymbol{K}\left(\tilde{\mathbf{H}}^{\prime}\right)$, we get

$$
\boldsymbol{K}\left(\mathbf{H}^{\prime}+\lambda \tilde{\mathbf{H}}^{\prime}\right)(\mathbf{p}) \leqq\left[\frac{h^{2} \boldsymbol{K}\left(\mathbf{H}^{\prime}\right)+\lambda \tilde{h}^{2} \boldsymbol{K}\left(\tilde{\mathbf{H}}^{\prime}\right)}{(h+\lambda \tilde{h})^{2}}\right](\mathbf{p})
$$

By hypothesis, there exists a negative constant $-K_{3}$ such that $\boldsymbol{K}(\tilde{\mathbf{H}})(\mathbf{p}) \leqq-K_{3}$. By the well-known decreasing property of hol. sect. curvature, we also have $\boldsymbol{K}\left(\tilde{\mathbf{H}}^{\prime}\right)(\mathbf{p}) \leqq \boldsymbol{K}(\tilde{\mathbf{H}})(\mathbf{p}) \leqq-K_{3}$. Similarly, $\boldsymbol{K}\left(\mathbf{H}^{\prime}\right)(\mathbf{p}) \leqq K_{4}$, for some positive constant $K_{4}$. Hence:

$$
\begin{aligned}
\boldsymbol{K}\left(\mathbf{H}^{\prime}+\lambda \tilde{\mathbf{H}}^{\prime}\right)(\mathbf{p}) & \leqq\left[\frac{h^{2} K_{4}-\lambda \tilde{h}^{2} K_{3}}{(h+\lambda \tilde{h})^{2}}\right](\mathbf{p}) \\
& =\left(\frac{h}{h+\lambda \tilde{h}}\right)^{2}\left(K_{4}-\lambda K_{3} \frac{\tilde{h}^{2}}{h^{2}}\right)(\mathbf{p}) \\
& =\left(\frac{h}{h+\lambda \tilde{h}}\right)^{2}\left(K_{4}-\lambda K_{3} \frac{\tilde{\mathbf{H}}\left(\frac{\partial}{\partial z}, \frac{\partial}{\partial z}\right)}{\mathbf{H}\left(\frac{\partial}{\partial z}, \frac{\partial}{\partial z}\right)}\right)(\mathbf{p}) .
\end{aligned}
$$

Since both the terms $\mathbf{H}\left(\frac{\partial}{\partial z}, \frac{\partial}{\partial z}\right), \tilde{\mathbf{H}}\left(\frac{\partial}{\partial z}, \frac{\partial}{\partial z}\right)$ are quadratic in $\frac{\partial}{\partial z}$, for the purpose of evaluating the fraction it suffices to take $\frac{\partial}{\partial z}$ so that $\mathbf{H}\left(\frac{\partial}{\partial z}, \frac{\partial}{\partial z}\right)(\mathbf{p})=1$. Since 
the unit sphere (relative to $\mathbf{H}$ ) is compact, clearly there exists a positive constant $K_{5}$ independent of $\lambda$ and $t=\frac{\partial}{\partial z}$, such that:

$$
K_{5} \leqq \frac{\tilde{\mathbf{H}}\left(\frac{\partial}{\partial z}, \frac{\partial}{\partial z}\right)}{\mathbf{H}\left(\frac{\partial}{\partial z}, \frac{\partial}{\partial z}\right)}(\mathbf{p})
$$

So we have:

$$
\boldsymbol{K}(\mathbf{H}+\lambda \tilde{\mathbf{H}}, t)=\boldsymbol{K}\left(\mathbf{H}^{\prime}+\lambda \tilde{\mathbf{H}}^{\prime}\right)(\mathbf{p}) \leqq\left(\frac{h}{h+\lambda \tilde{h}}\right)^{2}(\mathbf{p}) \cdot\left(K_{4}-\lambda K_{3} K_{5}\right) .
$$

So the Lemma follows if we choose $\lambda>\frac{K_{4}}{K_{3} K_{5}}$.

Remark. With hypothesis as in Lemma 4 and using the notation of the preceding proof, suppose that $\boldsymbol{K}\left(\mathbf{H}+\lambda_{1} \tilde{\mathbf{H}}\right) \leqq-K_{6}$, then using Lemma 2, we have, for $\lambda$ $>\lambda_{1}$,

$$
\boldsymbol{K}(\mathbf{H}+\lambda \tilde{\mathbf{H}})=\boldsymbol{K}\left(\mathbf{H}+\lambda_{1} \tilde{\mathbf{H}}+\left(\lambda-\lambda_{1}\right) \tilde{\mathbf{H}}\right) \leqq-\frac{K_{6} K_{3}}{K_{6}\left(\lambda-\lambda_{1}\right)+K_{3}}
$$

So $\boldsymbol{K}(\mathbf{H}+\lambda \tilde{\mathbf{H}}) \leqq-\mathbf{O}\left(\lambda^{-1}\right)$. Using $\mathbf{R}_{\alpha \bar{\beta} \gamma \bar{\delta}}^{\lambda}$ to denote the coefficients of the curvature tensor corresponding to the metric $\mathbf{H}+\lambda \tilde{\mathbf{H}}$, then from (2), we have:

$$
\sum \mathbf{R}_{\alpha \bar{\beta} \gamma \bar{\delta}}^{\lambda} \zeta^{\alpha} \bar{\zeta}^{\beta} \zeta^{\gamma} \bar{\zeta}^{\alpha} \leqq-\mathbf{O}\left(\lambda^{-1}\right) \lambda^{2} \sum \zeta^{\alpha} \bar{\zeta}^{\alpha} \zeta^{\beta} \bar{\zeta}^{\beta}=-\mathbf{O}(\lambda) \sum \zeta^{\alpha} \overline{\zeta^{\alpha}} \zeta^{\beta} \bar{\zeta}^{\beta} .
$$

\section{Construction of a Hermitian Form $\Phi$ on the Total Space $X$}

Under the assumptions of Theorem 1, Suppose $\left\{G_{t}\right\}$ is a smooth family of hermitian metrics with neg. hol. sect. curvature defined on each fiber, let $\varphi_{t}$ be the Hermitian form associated to the metric $G_{t}$. Fix $\tilde{\mathbf{G}}$ a Hermitian metric on $\mathbf{X}$. Now we want to construct a $(1,1)$-form $\Phi$ on $\mathbf{X}$, such that $\Phi$ restricted to each fibre $\pi^{-1}(t)$ is equal to $\varphi_{t}$. Let $Z_{1}$ and $Z_{2}$ be vector fields on $\mathbf{X}$ of type $(1,0)$ and $(0,1)$ respectively. At the point $\mathbf{p} \in \pi^{-1}(t)$, define:

$$
\Phi\left(Z_{1}, Z_{2}\right)(\mathbf{p}) \equiv \varphi_{t}\left(\operatorname{proj}_{\tilde{\mathbf{G}}} Z_{1}(\mathbf{p}), \operatorname{proj}_{\tilde{\mathbf{G}}} Z_{2}(\mathbf{p})\right)
$$

where $\operatorname{proj}_{\tilde{G}}$ is the projection onto the fibre direction with respect to the metric $\widetilde{G}$. Then clearly $\Phi$ is a $\mathscr{C}^{\infty}$, Hermitian $(1,1)$-form defined on $\mathbf{X}$, and $\Phi$ restricted to each fibre is equal to $\varphi_{t}$ which is positive definite.

Now, let us consider the case of Corollary 1, namely, each fibre has negative first Chern class and the canonical Kähler-Einstein metric $H_{t}$ on each fibre 
$\mathbf{X}_{t} \equiv \pi^{-1}(t)$ has neg. hol. sect. curvature. By Koiso [11], $\left\{H_{t}\right\}$ is a smooth family of Kähler metrics. So the preceding construction can now be applied to produce a global Hermitian $(1,1)$ form $\Phi$ on $\mathbf{X}$.

\section{Proof of Theorem 1}

Suppose that $\omega_{\mathbf{Y}}$ is the metric in $\mathbf{Y}$ that gives neg. hol. sect. curvature. We want to consider the form $\Psi_{\lambda} \equiv \Phi+\lambda \pi^{*}\left(\omega_{\mathbf{Y}}\right)$ with $\lambda$ a positive constant. It is obvious that when $\lambda$ is large enough, $\Psi_{\lambda}$ is a positive definite Hermitian $(1,1)$ form defined on $\mathbf{X}$. (Compare to the matrix example discussed in Sect. 2). By identifying this form with the Hermitian metric it defines, we want to show that this form will have neg. hol. sect, curvature on $\mathbf{X}$, when $\lambda$ is large enough.

Let $\mathbf{p}$ be a point in $\mathbf{X}$, we can assume that $\mathbf{p}$ lies in the fibre $\mathbf{X}_{0}$. Since $\pi$ is of maximal rank everywhere, locally there is a neighborhood $\mathbf{U}$ of $\mathbf{p}$ such that $\mathbf{U}=\mathbf{V} \times \mathbf{W}$, where $\mathbf{V}$ is a neighborhood of $\pi(\mathbf{p})$ in $\mathbf{Y}$, and $\mathbf{W}$ is a neighborhood of $\mathbf{p}$ in the fibre $\mathbf{X}_{\mathbf{0}}$. We may assume $\mathbf{V}, \mathbf{W}$ are co-ordinate neighborhoods, so there exist co-ordinates $\left\{z^{s+1}, \ldots, z^{n}\right\}$ in $\mathbf{V}$ and $\left\{z^{1}, z^{2}, \ldots, z^{s}\right\}$ in $\mathbf{W}$. Then $\left\{z^{1}, z^{2}, \ldots, z^{n}\right\}$ is a co-ordinate system around $\mathbf{p}$ in $\mathbf{U}$. For easier calculation, we will choose $\left\{z^{s+1}, \ldots, z^{n}\right\}$ so that $\frac{\partial}{\partial z^{s+1}}, \ldots, \frac{\partial}{\partial z^{n}}$ are orthonormal at $\pi(\mathbf{p})$ with respect to the metric $\omega_{\mathbf{y}}$. Let

$$
\begin{gathered}
\Phi=\sum_{i, j=1}^{n} g_{i \bar{j}} d z^{i} d \bar{z}^{j}, \\
\omega_{\mathbf{Y}}=\sum_{\alpha, \beta=s+1}^{n} \tilde{g}_{\alpha \bar{\beta}}\left(z^{s+1}, \ldots, z^{n}\right) d z^{\alpha} d \bar{z}^{\beta}, \quad \text { with } \tilde{g}_{\alpha \bar{\beta}}(\mathbf{p})=\delta_{\alpha \bar{\beta}}
\end{gathered}
$$

then the Hermitian metric $\Psi_{\lambda}$ with respect to $\left\{z^{1}, z^{2}, \ldots, z^{n}\right\}$ is given by:

$$
\begin{aligned}
\sum_{i, j=1}^{n} h_{i \bar{j}} d z^{i} d \bar{z}^{j}= & \sum_{i, j=1}^{s} g_{i \bar{j}} d z^{i} d \bar{z}^{j}+\sum_{i=1}^{s} \sum_{\alpha} g_{i \bar{\alpha}} d z^{i} d \bar{z}^{\alpha} \\
& +\sum_{j=1}^{s} \sum_{\beta} g_{\beta \bar{j}} d z^{\beta} d \bar{z}^{j}+\sum_{\alpha, \beta}\left(g_{\alpha \bar{\beta}}+\lambda \tilde{g}_{\alpha \bar{\beta}}\right) d z^{\alpha} d \bar{z}^{\beta}
\end{aligned}
$$

where $\alpha, \beta$ run from $s+1$ to $n$. Denote by $\mathbf{A}$ the $s \times s$ matrix with coefficients $\left(g_{a b}(\mathbf{p})\right)$, with $a, b \leqq s$, and denote by $\mathbf{A}_{a b}$ the $(a, b)^{\text {th }}$ cofactor of the matrix $\mathbf{A}$. Then by direct calculation it is easy to see that:

$$
h^{a \bar{b}}(\mathbf{p})=\frac{\lambda^{n-s} \operatorname{det} \mathbf{A}_{a b}+\mathbf{O}\left(\lambda^{n-s-1}\right)}{\lambda^{n-s} \operatorname{det} \mathbf{A}+\mathbf{O}\left(\lambda^{n-s-1}\right)}, \quad h^{\times \not z}(\mathbf{p})=\frac{\lambda^{n-s-1} \operatorname{det} \mathbf{A}+\mathbf{O}\left(\lambda^{n-s-2}\right)}{\lambda^{n-s} \operatorname{det} \mathbf{A}+\mathbf{O}\left(\lambda^{n-s-1}\right)}
$$

$h^{a \bar{\chi}}(\mathbf{p})=\mathbf{O}\left(\lambda^{-1}\right), h^{\chi \bar{b}}(\mathbf{p})=\mathbf{O}\left(\lambda^{-1}\right), h^{\chi \bar{\gamma}}(\mathbf{p})=\mathbf{O}\left(\lambda^{-2}\right)$, for $a, b \leqq s$, and $\chi, \eta \geqq s+1$ with $\chi \neq \eta$. 
Now. let us check the conditions of Lemma 1. First look at Condition (i): Substitute (9) into formula (1), we have, when $i, j, k, l \leqq s$,

$$
\begin{aligned}
\mathbf{R}_{i \overline{i j k} \bar{l}}(\mathbf{p}) & =-\left.\frac{\partial^{2} g_{i \bar{j}}}{\partial z^{k} \partial \bar{z}^{\bar{z}}}\right|_{\mathbf{p}}+\left.\left(\sum_{a, b=1}^{s} h^{b a} \frac{\partial g_{i \bar{a}}}{\partial z^{k}} \frac{\partial g_{b \bar{j}}}{\partial \bar{z}^{\bar{j}}}\right)\right|_{\mathbf{p}}+\left.\left(\sum_{\substack{u, v \\
\text { not both } \leqq s}} h^{v \bar{a}} \frac{\partial g_{i \bar{a}}}{\partial z^{k}} \frac{\partial g_{v \bar{j}}}{\partial \bar{z}^{k}}\right)\right|_{\mathbf{p}} \\
& =-\left.\frac{\partial^{2} g_{i \bar{j}}}{\partial z^{k} \partial \bar{z}^{l}}\right|_{\mathbf{p}}+\left.\left(\sum_{a, b=1}^{s} h^{b \bar{a}} \frac{\partial g_{i \bar{a}}}{\partial z^{k}} \frac{\partial g_{b \bar{j}}}{\partial \bar{z}^{l}}\right)\right|_{\mathbf{p}}+\mathbf{O}\left(\lambda^{-1}\right) .
\end{aligned}
$$

Observe that $\lim _{\lambda \rightarrow \infty} h^{b \bar{a}}(\mathbf{p})=\frac{\operatorname{det} \mathbf{A}_{b a}}{\operatorname{det} \mathbf{A}}$ and furthermore

$$
\sum_{i, j, k, l=1}^{s}\left(-\left.\frac{\partial^{2} g_{i j}}{\partial z^{k} \partial \bar{z}^{l}}\right|_{\mathbf{p}}+\left.\sum_{a, b=1}^{s} \frac{\operatorname{det} \mathbf{A}_{b a}}{\operatorname{det} \mathbf{A}} \frac{\partial g_{i \bar{a}}}{\partial z^{k}} \frac{\partial g_{b \bar{j}}}{\partial \bar{z}^{l}}\right|_{\mathbf{p}}\right) \zeta^{i} \zeta^{j} \zeta^{k} \zeta
$$

is the numerator of the hol. sect. curvature of the induced metric on each fibre and which by assumption is bounded by a negative constant. It follows that if $\lambda$ is large enough, condition (i) is satisfied.

Using (9) and the fact that $\tilde{g}_{\alpha \bar{\beta}}$ is a function of $z^{s+1}, \ldots, z^{n}$ only, (this fact is similar to the vanishing of the first column and first row in the example of the matrices mentioned in Sect. 2), while

$$
-\left.\frac{\partial^{2} h_{i \bar{j}}}{\partial z^{u} \partial \bar{z}^{l}}\right|_{\mathbf{p}},\left.\quad \frac{\partial h_{i \bar{\beta}}}{\partial z^{u}}\right|_{\mathbf{p}}
$$

are both independent of $\lambda$, when $u \leqq s$, it is easy to check that $\left|\mathbf{R}_{i j k} \bar{l}(\mathbf{p})\right| \leqq \mathbf{O}(1)$ whenever $\min (i, j, k, l) \leqq s$. Hence the second condition is satisfied when $\lambda$ is large enough.

As for condition (iii), we have to estimate

$$
\sum_{\alpha, \beta, \gamma, \delta=s+1}^{\mathbf{n}} \mathbf{R}_{\alpha \bar{\beta} \gamma \bar{\delta}}(\mathbf{p}) \zeta^{\alpha} \bar{\zeta}^{\beta} \zeta^{\gamma} \bar{\zeta}^{\delta} .
$$

By definition it is equal to:

$$
=\sum_{\alpha, \beta, \gamma, \delta=s+1}^{n}\left(-\left.\frac{\partial^{2} g_{\alpha \bar{\beta}}+\lambda \tilde{g}_{\alpha \bar{\beta}}}{\partial z^{\gamma} \partial \bar{z}^{\delta}}\right|_{\mathbf{p}}+\left.\sum_{u, v=1}^{n} h^{v \bar{a}}(\mathbf{p}) \frac{\partial h_{\alpha \bar{u}}}{\partial z^{\gamma}} \frac{\partial h_{\bar{v} \beta}}{\partial \bar{z}^{\bar{\delta}}}\right|_{\mathbf{p}}\right) \zeta^{\alpha} \bar{\zeta}^{\beta} \zeta^{\gamma} \bar{\zeta}^{\delta} .
$$

Again using (9), we have:

$$
\begin{aligned}
& \sum_{\alpha, \beta, \gamma, \delta=s+1}^{n} \mathbf{R}_{\alpha \bar{\beta} \gamma \bar{\delta}}(\mathbf{p}) \zeta^{\alpha} \bar{\zeta}^{\beta} \zeta^{\gamma} \bar{\zeta}^{\delta}=\sum_{\alpha, \beta, \gamma, \delta=s+1}^{n}\left(-\left.\frac{\partial^{2} \mathbf{g}_{\alpha \bar{\beta}}+\lambda \tilde{\mathbf{g}}_{\alpha \bar{\beta}}}{\partial z^{\gamma} \partial \bar{z}^{\delta}}\right|_{\mathbf{p}}\right. \\
& \left.\quad+\left.\sum_{\chi=s+1} h^{\chi \bar{\chi}}(\mathbf{p}) \lambda^{2} \frac{\partial \tilde{\mathbf{g}}_{\alpha \chi}}{\partial z^{\gamma}} \frac{\partial \tilde{\mathbf{g}}_{\chi \bar{\beta}}}{\partial \bar{z}^{\delta}}\right|_{\mathbf{p}}+\mathbf{O}(1)\right) \zeta^{\alpha} \bar{\zeta}^{\beta} \zeta^{\gamma} \bar{\zeta}^{\delta} .
\end{aligned}
$$


Now consider a hypersurface $\mathbf{M}^{\prime}$ around $\mathbf{p}$ defined by $\left\{z^{1}=z^{2}=\ldots=z^{s}=0\right\}$. We would like to compare expression (10) with the hol. sect. curvature of the induced metric on $\mathbf{M}^{\prime}$. It is well known that the two differ by some positive terms, but in our case all these terms turn out to be of order $\lambda^{0}$, as we shall see.

Let $\mathbf{G}^{\prime}$ be the Hermitian metric on $\mathbf{M}^{\prime}$ induced by $\Phi$. Notice that $\pi^{*}\left(\omega_{\mathbf{Y}}\right)$ becomes a Hermitian metric on $\mathbf{M}^{\prime}$; let us denote it by $\tilde{\mathbf{G}}^{\prime}$. Then $\tilde{\mathbf{G}}^{\prime}$ has neg. hol. sect. curvature. Also we have:

$$
\mathbf{G}^{\prime}=\sum_{\alpha, \beta=s+1}^{n} g_{\alpha \bar{\beta}} d z^{\alpha} d \bar{z}^{\beta}, \quad \tilde{\mathbf{G}}^{\prime}=\sum_{\alpha, \beta=s+1}^{n} \tilde{g}_{\alpha \bar{\beta}} d z^{\alpha} d \bar{z}^{\beta} .
$$

Let

$$
\left.\Psi_{\lambda}\right|_{\mathbf{M}^{\prime}}=\mathbf{G}^{\prime}+\lambda \tilde{G}^{\prime}=\sum_{\alpha, \beta=s+1}^{n} k_{\alpha \bar{\beta}} d z^{\alpha} d \bar{z}^{\beta}
$$

And similar to (9), we have:

$$
\begin{aligned}
& k^{\chi \bar{\chi}}(\mathbf{p})=\frac{\lambda^{n-2}+\mathbf{O}\left(\lambda^{n-3}\right)}{\lambda^{n-1}+\mathbf{O}\left(\lambda^{n-2}\right)}, \\
& k^{\chi \bar{\eta}}(\mathbf{p})=\mathbf{O}\left(\lambda^{-2}\right) \quad \text { where } \chi, \eta \geqq s+1 \text { and } \chi \neq \eta .
\end{aligned}
$$

Using $\mathbf{R}_{\alpha \bar{\beta} \gamma \bar{\delta}}^{\prime}$ to denote the coefficients of the curvature tensor of the induced metric, then corresponding to (10), we have:

$$
\begin{aligned}
& \sum_{\alpha, \beta, \gamma, \delta=s+1}^{n} \mathbf{R}_{\alpha \bar{\beta} \gamma \bar{\delta}}^{\prime}(\mathbf{p}) \zeta^{\alpha} \bar{\zeta}^{\beta} \zeta^{\gamma} \bar{\zeta}^{\delta} \\
& =\sum_{\alpha, \beta, \gamma, \delta=s+1}^{n}\left(-\left.\frac{\partial^{2} g_{\alpha \bar{\beta}}+\lambda \tilde{g}_{\alpha \bar{\beta}}}{\partial z^{\gamma} \partial \bar{z}^{\delta}}\right|_{\mathbf{p}}+\left.\sum_{\chi=s+1} k^{\chi \bar{\chi}}(\mathbf{p}) \lambda^{2} \frac{\partial \tilde{g}_{\alpha \bar{\chi}}}{\partial z^{\gamma}} \frac{\partial \tilde{g}_{\chi \bar{\beta}}}{\partial \bar{z}^{\delta}}\right|_{\mathbf{p}}\right. \\
& \left.\quad+\mathbf{O}\left(\lambda^{-2}\right)+\mathbf{O}\left(\lambda^{-1}\right)+\mathbf{O}(1)\right) \zeta^{\alpha \bar{\zeta} \zeta^{\gamma} \bar{\zeta} \delta} .
\end{aligned}
$$

But since $\lambda^{2} k^{\chi \bar{x}}(\mathbf{p})-\lambda^{2} h^{\chi \bar{x}}(\mathbf{p})=\mathbf{O}(1)$. Compare (10) and (12), we have:

$$
\sum_{\alpha, \beta, \gamma, \delta=2}^{n} \mathbf{R}_{\alpha \bar{\beta} \gamma \bar{\delta}}(\mathbf{p}) \zeta^{\alpha} \bar{\zeta}^{\beta} \zeta^{\gamma \overline{\zeta^{\delta}}}=\sum_{\alpha, \beta, \gamma, \delta=2}^{n}\left(\mathbf{R}_{\alpha \bar{\beta} \gamma \bar{\delta}}^{\prime}(\mathbf{p})+\mathbf{O}(1)\right) \zeta^{\alpha} \overline{\zeta^{\beta}} \zeta^{\gamma} \overline{\zeta^{\delta}} .
$$

By inequality (8), the last expression can be made arbitrary negative by increasing $\lambda$. Hence by Lemma 1 , if $\lambda$ is large enough, the Hermitian metric, $\Psi_{\lambda}$, will have neg. hol. sect. curvature at point p. Hence there exists a neighborhood $\mathscr{U}_{\mathbf{p}, \lambda}$ around p such that $\Psi_{\lambda}$ has neg. hol. sect. curvature at all points in $\mathscr{U}_{\mathbf{p}, \lambda}$.

Lemma 5. Let $\Phi+\lambda \pi^{*}\left(\omega_{\mathbf{Y}}\right)$ have neg. hol. sect. curvature in $\mathscr{U}_{\mathbf{p}, \lambda}$. If $\lambda_{1}>\lambda$, then $\Phi+\lambda_{1} \pi^{*}\left(\omega_{\mathbf{Y}}\right)$ will also have neg. hol. sect. curvature at all points in $\mathscr{U}_{\mathbf{p}, \lambda}$.

Proof. Let $\mathbf{q} \in \mathscr{U}_{\mathbf{p}, \lambda}$, let $\boldsymbol{K}_{\lambda_{1}}(\vec{v})$ denote the hol. sect. curvature of the metric $\Phi$ $+\lambda_{1} \pi^{*}\left(\boldsymbol{\omega}_{\mathbf{Y}}\right)$ in the direction $\vec{v}$ at $\mathbf{q}$. 
Case 1. If $\vec{v}$ is not tangent to the fibre, then $\pi^{*}\left(\omega_{\mathbf{Y}}\right)(\vec{v}, \vec{v})>0$. Thus $\vec{v}$ is tangent to a locally defined complex manifold $Z$ transversal to the fibres so that $\pi^{*}\left(\boldsymbol{\omega}_{\mathbf{Y}}\right)$ restricted to $Z$ is a Hermitian metric. Since

$$
\Phi+\lambda_{1} \pi^{*}\left(\boldsymbol{\omega}_{\mathbf{Y}}\right)=\left(\Phi+\lambda \pi^{*}\left(\boldsymbol{\omega}_{\mathbf{Y}}\right)\right)+\left(\lambda_{1}-\lambda\right) \pi^{*}\left(\boldsymbol{\omega}_{\mathbf{Y}}\right),
$$

applying Lemma 2, we can conclude that $\boldsymbol{K}_{\lambda_{1}}(\vec{v})$ is negative.

Case 2. If $\vec{v}$ is tangent to the fibre, then there exists a coordinate system $\left\{z^{1}, z^{2}, \ldots, z^{n}\right\}$, such that $\vec{v}=\frac{\partial}{\partial z^{1}}(\mathbf{q})$. Then for some positive constants $K_{7}, K_{8}$, we have:

$$
\begin{gathered}
\boldsymbol{K}_{\lambda_{1}}(\vec{v})=\left(-\left.\frac{\partial^{2} g_{1 \bar{i}}}{\partial z^{1} \partial \bar{z}^{1}}\right|_{\mathbf{q}}+\left.\sum_{i, j}\left(\left(h^{\lambda_{1}}\right)^{j \bar{i}} \frac{\partial g_{1 \bar{i}}}{\partial z^{1}} \frac{\partial g_{j \bar{i}}}{\partial \bar{z}^{1}}\right)\right|_{\mathbf{q}}\right) \cdot K_{7}, \\
\boldsymbol{K}_{\lambda}(\vec{v})=\left(-\left.\frac{\partial^{2} \boldsymbol{g}_{1 \overline{1}}}{\partial z^{1} \partial \bar{z}^{1}}\right|_{\mathbf{q}}+\left.\sum_{i, j}\left(\left(h^{\lambda}\right)^{j i \bar{i}} \frac{\partial g_{1 \bar{i}}}{\partial z^{1}} \frac{\partial \boldsymbol{g}_{j \overline{1}}}{\partial \bar{z}^{1}}\right)\right|_{\mathbf{q}}\right) \cdot K_{8},
\end{gathered}
$$

where $\left(h^{\lambda_{1}}\right)_{i j}$ and $\left(h^{\lambda}\right)_{i \bar{j}}$ are the coefficients of the metrics $\Phi+\lambda_{1} \pi^{*}\left(\boldsymbol{\omega}_{\mathbf{Y}}\right)$ and $\Phi$ $+\lambda \pi^{*}\left(\boldsymbol{\omega}_{\mathbf{Y}}\right)$ respectively. By Lemma 3 ,

$$
\boldsymbol{K}_{\lambda_{1}}(\vec{v}) / K_{7}-K_{\lambda}(\vec{v}) / K_{8} \leqq 0, \quad \text { if } \lambda_{1} \geqq \lambda .
$$

So again we have $\boldsymbol{K}_{\lambda_{1}}(\vec{v})$ is negative.

Apply the construction to every point, then using Lemma 5 and a compactness argument, we can conclude that $\Phi+\lambda \pi^{*}\left(\boldsymbol{\omega}_{\mathbf{Y}}\right)$ has neg. hol. sect. curvature at all points in $\mathbf{X}$, if $\lambda$ is large enough. This completes the proof of Theorem 1.

Corollary 1 follows immediately from Theorem 1 and the construction of the form $\Phi$ in Sect. 4.

In the case when the fibre is of dimension 1 , then $\mathbf{X}$ is hyperbolic implies that each fibre must have genus $\geqq 2$, and Corollary 2 follows from Corollary 1 . However, Corollary 2 can also be proved directly without using the arguments of Koiso, see Cheung [3].

\section{Kodaira's Surfaces of General Type}

A Kodaira surface $\mathbf{M}$ can be considered as a $m$-sheeted ramified covering of $\mathbf{W}=\mathbf{R} \times \mathbf{S}, m \geqq 2$, with branch loci $\Gamma$ and $\Gamma^{*}$, where $\mathbf{R}, \mathbf{S}$ are some Riemann surfaces of genus $\geqq 2, \Gamma$ and $\Gamma^{*}$ are the graphs of some holomorphic maps from $\mathbf{S} \rightarrow \mathbf{R}$. (For the actual construction, the reader can refer to Kodaira [9], or [1], p. 167). Following the notation of Kodaira [9], we have that if we let $\mu$ be the covering map of $\mathbf{M}$ onto $\mathbf{W}=\mathbf{R} \times \mathbf{S}, p_{2}$ be the second projection of $\mathbf{W}=\mathbf{R} \times \mathbf{S}$ onto $\mathbf{S}$, then the inverse images of the branch loci are $\boldsymbol{\Delta} \equiv \mu^{-1}(\boldsymbol{\Gamma})$, $\Delta^{*} \equiv \mu^{-1}\left(\Gamma^{*}\right), \Delta$ and $\Delta^{*}$ are biholomorphic to $\Gamma$ and $\Gamma^{*}$ respectively.

Consider the product metric in $\mathbf{W}=\mathbf{R} \times \mathbf{S}: \omega_{\mathbf{W}}=\omega_{\mathbf{R}}+\omega_{\mathbf{S}}$, where $\omega_{\mathbf{R}}, \omega_{\mathbf{S}}$ are the metrics in $\mathbf{R}$ and $\mathbf{S}$ with curvature -1 , then a standard computation shows: 
(13) $\mu^{*}\left(\omega_{\mathbf{w}}\right)$ is a Kähler metric defined in $\mathbf{M} \backslash\left(\Delta \cup \Delta^{*}\right)$ with hol. sect. curvature $\leqq-\frac{1}{2}$.

So in order to get a Kähler metric on $\mathbf{M}$, we have to construct a positive-definite closed form around $\Delta$ and $\Delta^{*}$.

Consider a point $\mathbf{a}$ in $\boldsymbol{\Delta}$ (with similar considerations for $\boldsymbol{\Delta}^{*}$ ), suppose $\left\{\boldsymbol{r}_{a}, s_{a}\right\}$ are the product coordinates at $\mu(\mathbf{a}) \in \mathbf{R} \times \mathbf{S}$, then if $f_{a}$ is a defining function of $\Gamma$ around $\mu(\mathbf{a}),\left\{f_{a}, s_{a}\right\}$ will be a local coordinate system around $\mu(\mathbf{a})$. Let $g_{a}$ be the defining function for $\Delta$ at a, such that $\left(g_{a}\right)^{m}=\mu^{*}\left(f_{a}\right)$. Since $\mathbf{M}$ is a $m$-sheeted cover of $\mathbf{W},\left\{g_{a}, \mu^{*}\left(s_{a}\right)\right\}$ is a coordinate system around $\mathbf{a}$.

We notice that if $z$ is a coordinate function, then $\sqrt{-1} \partial \bar{\partial}\left(z \bar{z}+\frac{1}{4}(z \bar{z})^{2}\right)$ gives a positive form with negative curvature, so in our construction we would consider the form $\sqrt{-1} \partial \bar{\partial}\left(g_{a} \bar{g}_{a}+\frac{1}{4}\left(g_{a} \bar{g}_{a}\right)^{2}\right)$. But since $g_{a}$ is not defined outside a neighbourhood of a, we have to multiply it with some cut-off functions. These take care the " $g_{a}$ " direction. Then along the $\mu^{*}\left(s_{a}\right)$ direction, we consider the pull back of the metric of $\mathbf{S}$, namely, $\mu^{*} \mathrm{p}_{2}^{*}\left(\omega_{\mathrm{S}}\right)$. The constructions are carried out in the next section.

\section{A Kähler Form $\Phi_{c, k}$ in $M$ with Neg. Hol. Sect. Curvature}

Following Sect. 6 , at each point $\mathbf{a} \in \Delta$ or $\Delta^{*}$, we can choose $\varepsilon_{a}, \delta_{a}$ small enough, such that $\mathscr{U}_{a}=\left\{\left|g_{a}\right|^{2}<\varepsilon_{a}\right\} \cap\left\{\left|\mu^{*}\left(s_{a}\right)\right|\left\langle\delta_{a}\right\}\right.$ is a well-defined neighbourhood of a in $\mathbf{M}$.

Let $\mathscr{U}_{a / 2}=\left\{\left|g_{a}\right|^{2}<\frac{1}{2} \varepsilon_{a}\right\} \cap\left\{\left|\mu^{*}\left(s_{a}\right)\right|<\frac{1}{2} \delta_{a}\right\}$, then since $\boldsymbol{A}$ and $\boldsymbol{A}^{*}$ are compact, there exists $\left\{a_{1}, \ldots, a_{n}\right\}$ such that $\Delta \cup \Delta^{*} \subset \bigcup_{i=1}^{n} \mathscr{U}_{a_{i} / 2}$. Define the cut-off functions:

$$
\rho_{i}: \mathbb{R}^{+} \cup\{0\} \rightarrow \mathbb{R}^{+} \cup\{0\}
$$

such that $\rho_{i} \equiv 1$ when $x \leqq \frac{1}{2} \varepsilon_{a_{i}}$ and is identical to 0 when $x \geqq \varepsilon_{a_{i}}$

$$
\chi_{i}: \mathbb{C} \rightarrow \mathbb{R}^{+} \cup\{0\}
$$

such that $\chi_{i}$ is rotationally symmetric and is identical to 1 inside the disc $D\left(\frac{1}{2} \delta_{a_{i}}\right)$ and identical to 0 outside the disc $D\left(\delta_{a_{i}}\right)$.

If no confusion is possible, we will denote the index " $a_{i}$ " also by " $i$ ". Now with these cut-off functions, $\rho_{i}\left(g_{i} \bar{g}_{i}\right) \cdot\left(g_{i} \bar{g}_{i}+\frac{1}{4}\left(g_{i} \bar{g}_{i}\right)^{2}\right) \cdot \chi_{i}\left(s_{i}\left(p_{2} \circ \mu\right)\right)$ is a $\mathscr{C}^{\infty}$ function defined in $\mathbf{M}$, with compact support inside $\mathscr{U}_{a_{i}}$ and is identical to $g_{i} \bar{g}_{i}$ $+\frac{1}{4}\left(g_{i} \bar{g}_{i}\right)^{2}$ inside $\mathscr{U}_{a_{i} / 2}$.

Hereafter, we shall identify a Hermitian metric $\sum g_{i j} d z^{i} d \bar{z}^{j}$ with its Kähler form $\sqrt{-1} \sum g_{i j} d z^{i} \wedge d \bar{z}^{j}$. Define

$$
\tilde{\omega}_{k} \equiv \sqrt{-1} \sum_{i=1}^{n} \partial \bar{\partial}\left(\rho_{i}\left(g_{i} \bar{g}_{i}\right) \cdot\left(g_{i} \bar{g}_{i}+\frac{1}{4}\left(g_{i} \bar{g}_{i}\right)^{2}\right) \cdot \chi_{i}\left(s_{i}\left(p_{2} \circ \mu\right)\right)\right)+k \mu^{*} p_{2}^{*}\left(\omega_{\mathrm{S}}\right)
$$


and let $\Phi_{c, k} \equiv \mu^{*} \omega_{\mathbb{W}}+c \tilde{\omega}_{k}$ where $c, k$ are positive numbers to be determined later.

Now consider the point $a_{1}$. Without losing generality we can assume that $a_{1} \in \Delta$. Let $\left\{z^{1}=g_{1}, z^{2}=\mu^{*} p_{2}^{*}\left(s_{1}\right)\right\}$ be the local coordinates inside $\mathscr{U}_{a_{1} / 2}$, then $g_{i}=h_{i} z^{1}$, where $h_{i}$ is a non-zero holomorphic function defined in $\mathscr{U}_{a_{i}} \cap \mathscr{U}_{a_{1}}$, and $\mu^{*} p_{2}^{*}\left(s_{i}\right)=q_{i}\left(z^{2}\right)$, where $q_{i}$ is biholomorphic inside the region $\left\{\left|s_{1}\left(p_{2} \circ \mu\right)\right|\right.$ $\left.<\delta_{a_{1}}\right\} \cap\left\{\left|s_{i}\left(p_{2} \circ \mu\right)\right|<\delta_{a_{3}}\right\}$. Let $\mu^{*} p_{2}^{*}\left(\omega_{\mathbf{s}}\right)=\sqrt{-1} \tilde{p}\left(z^{2}\right) d z^{2} \wedge d \bar{z}^{2}$ in local co-ordinates. Then using the fact that $\rho_{i}$ is $\equiv 1$ near the branch locus $\left(\Delta\right.$ or $\left.\Delta^{*}\right)$, and $z^{1}=0$ along the locus, by a simple computation we get:

$$
\left.\tilde{\boldsymbol{\omega}}_{\boldsymbol{k}}\right|_{\mathscr{u}_{a_{1} / 2 \cap \tilde{\mathbf{A}}}}=\sqrt{-1}\left(d z^{1} \wedge d \bar{z}^{1}+\sum_{i \neq 1}^{n} \chi_{i}\left(q_{i}\left(z_{2}\right)\right) h_{i} \bar{h}_{i} d z^{1} \wedge d \bar{z}^{1}+k \tilde{p}\left(z_{2}\right) d z^{2} \wedge d \bar{z}^{2}\right)
$$

which is of course positive definite. Do the same to the other $\mathscr{U}_{a_{i} / 2}$. We have $\tilde{\omega}_{k}$ is positive definite on $\Delta \cup \Delta^{*}$. Therefore there exists a neighborhood $\mathscr{V}_{k}$ of $\boldsymbol{\Delta} \cup \boldsymbol{A}^{*}$ such that $\tilde{\boldsymbol{\omega}}_{k}$ is positive definite in $\mathscr{V}_{k}$. On the other hand, $\mu^{*} \omega_{\mathrm{W}}$ is positive semi-definite in $\mathbf{M}$. Therefore $\Phi_{c, k}$, which is equal to $\mu^{*} \omega_{\mathbf{w}}+c \tilde{\omega}_{k}$, is positive definite in $\mathscr{V}_{k}$ for any $c$. Now, in the compact region $\mathbf{M} \backslash \mathscr{V}_{k}$ :

$$
\lim _{c \rightarrow 0} \Phi_{c, k}=\mu^{*} \omega_{\mathrm{w}} \quad \text { which is positive definite. }
$$

So if we choose $c$ small enough, $\Phi_{c, k}$ will also be positive definite in $\mathbf{M} \backslash \mathscr{V}_{k}$. Therefore we can conclude that $\Phi_{c, k}$ is a Kähler form defined on $\mathbf{M}$ and is positive definite, when $c$ is small enough.

Next step is to check the curvature of $\Phi_{c, k}$. Since $\tilde{\omega}_{k}$ defines a metric near $\boldsymbol{\Delta} \cup \boldsymbol{\Delta}^{*}$, we want to apply Lemma 1 to check its hol. sect. curvature there. Let $\tilde{\omega}_{k}=\sqrt{-1} \sum G_{i \bar{j}} d z^{i} \wedge d \bar{z}^{j}$ in $\mathscr{U}_{a_{i} / 2}$, then the $G_{i \bar{j}}$ terms can be calculated using formula (14). (The calculation is actually much simpler than it looks, because $h_{j}$ is holomorphic, $\chi_{j}$ is a function of $z^{2}$ only, $\rho_{j}$ is $\equiv 1$ near $\Delta$ or $\Delta^{*}$, a lot of terms will vanish.) In particular along $\Delta \cap \mathscr{U}_{a_{1 / 2}}$, that is when $z^{1}=0$, we have:

$$
\begin{gathered}
G_{1 \overline{1}}=1+\sum_{j \neq 1} h_{j} \bar{h}_{j} \chi_{j}\left(q_{j}\left(z^{2}\right)\right), \quad G_{1 \overline{2}} \equiv 0, \quad G_{2 \overline{1}} \equiv 0, \quad G_{2 \bar{z}}=k \tilde{p}\left(z_{2}\right) ; \\
\frac{\partial G_{1 \overline{2}}}{\partial z^{2}}=\frac{\partial G_{1 \overline{2}}}{\partial \bar{z}^{2}}=\frac{\partial G_{2 \overline{1}}}{\partial z^{2}}=\frac{\partial G_{2 \overline{1}}}{\partial \bar{z}^{2}}=\frac{\partial G_{1 \overline{2}}}{\partial z^{1}}=\frac{\partial G_{2 \overline{2}}}{\partial z^{1}}=\frac{\partial G_{2 \overline{2}}}{\partial \bar{z}^{1}}=\frac{\partial G_{2 \overline{1}}}{\partial \bar{z}^{1}}=0 \\
\frac{\partial G_{2 \overline{2}}}{\partial z^{2}}=\frac{\partial}{\partial z^{2}} k \tilde{p}\left(z_{2}\right), \quad \frac{\partial G_{2 \overline{2}}}{\partial \bar{z}^{2}}=\frac{\partial}{\partial \bar{z}^{2}} k \tilde{p}\left(z^{2}\right) ;
\end{gathered}
$$

while all other $\frac{\partial G_{\beta \bar{\gamma}}}{\partial z^{\alpha}}$ terms are independent of $k$ and since they are well-defined inside $\mathscr{U}_{a_{1}}$, there exists a constant $K_{9}$, independent of $k$ such that

$$
\left|\frac{\partial G_{\beta \bar{\gamma}}}{\partial z^{\alpha}}\right|<K_{9} \quad \text { in } \quad \mathscr{U}_{a_{1 / 2}} \cap \boldsymbol{A} .
$$


Similarly, we have

$$
\left|\frac{\partial^{2} G_{\gamma \bar{\delta}}}{\partial z^{\alpha} \partial \bar{z}^{\bar{\beta}}}\right|<K_{9} \quad \text { in } \quad \mathscr{U}_{a_{1} / 2} \cap \Delta
$$

for some constant $K_{9}$ when $\gamma$ and $\delta$ are not both equal to 2 .

Now we can check the conditions for Lemma 1 along $\boldsymbol{\Delta} \cap \mathscr{U}_{a_{1} / 2}$ :

$\mathbf{R}_{\left.1 \overline{1} 1 \overline{1}\right|_{z^{1}=0}}=-\left.\frac{\partial^{2} G_{1 \overline{1}}}{\partial z^{1} \partial \bar{z}^{1}}\right|_{z^{1}=0}+\left.\frac{1}{G_{1 \overline{1}}} \frac{\partial G_{1 \overline{1}}}{\partial z^{1}} \frac{\partial G_{1 \overline{1}}}{\partial \bar{z}^{1}}\right|_{z^{1}=0}$

(all other terms vanish)

$$
\begin{aligned}
= & -1-\sum_{j \neq 1}\left(4 \frac{\partial h_{j}}{\partial z^{1}} \cdot \frac{\partial \bar{h}_{j}}{\partial \bar{z}^{1}} \cdot \chi_{j}\left(q_{j}\left(z^{2}\right)\right)+\left(h_{j} \bar{h}_{j}\right)^{2} \cdot \chi_{j}\left(q_{j}\left(z^{2}\right)\right)\right) \\
& +\frac{1}{1+\sum_{j \neq 1} h_{j} \bar{h}_{j} \chi_{j}\left(q_{j}\left(z^{2}\right)\right)}\left(2 \sum_{j \neq 1} \frac{\partial h_{j}}{\partial z^{1}} \bar{h}_{j} \chi_{j}\left(q_{j}\left(z^{2}\right)\right)\right)\left(2 \sum_{j \neq 1} \frac{\partial \bar{h}_{j}}{\partial \bar{z}^{1}} h_{j} \chi_{j}\left(q_{j}\left(z^{2}\right)\right)\right) \\
\leqq & -1-\sum_{j \neq 1}\left(h_{j} \bar{h}_{j}\right)^{2} \chi_{j}\left(q_{j}\left(z^{2}\right)\right) \leqq-1,
\end{aligned}
$$

$\left.\mathbf{R}_{2 \overline{2} 2 \overline{2}}\right|_{z^{1}=0}=-\left.\frac{\partial^{2} G_{2 \bar{z}}}{\partial z^{2} \partial \bar{z}^{2}}\right|_{z^{1}=0}+\left.\frac{1}{G_{2 \overline{2}}} \frac{\partial G_{2 \overline{2}}}{\partial z^{2}} \frac{\partial G_{2 \overline{2}}}{\partial \bar{z}^{2}}\right|_{z^{1}=0}$

(all other terms vanish)

$$
=-\frac{\partial^{2} k \tilde{p}\left(z^{2}\right)}{\partial z^{2} \partial \bar{z}^{2}}+\frac{k}{\tilde{p}\left(z^{2}\right)} \frac{\partial \tilde{p}\left(z^{2}\right)}{\partial z^{2}} \frac{\partial \tilde{p}\left(z^{2}\right)}{\partial \bar{z}^{2}}=-k\left(\tilde{p}\left(z^{2}\right)\right)^{2}
$$

(because $\tilde{p}$ is the metric coefficient corresponding to -1 curvature in $\mathbf{S}$ ).

By (15) and (16), all other coefficients of the curvature tensor are bounded inside $\mathscr{U}_{a_{1} / 2} \cap \Delta$ and independent of $k$.

By Lemma 1, if we choose $k_{1}$ large enough, then $\tilde{\omega}_{k_{1}}$ will have neg. hol. sect. curvature along $\mathscr{U}_{a_{1} / 2} \cap \Delta$. Similarly for other points $\left\{a_{2}, \ldots, a_{n}\right\}$. Let $\tilde{k}$ $=\max \left\{k_{1}, \ldots, k_{n}\right\}$, then $\tilde{\omega}_{\tilde{k}}$ will have neg. hol. sect. curvature at all points on $\boldsymbol{\Delta} \cup \boldsymbol{A}^{*}$. Therefore there exists a neighborhood $\mathscr{U}_{\tilde{k}}$ of $\boldsymbol{\Delta} \cup \boldsymbol{\Delta}^{*}$, which is independent of $c$, such that the hol. sect. curvature $K\left(\tilde{\omega}_{\tilde{k}}\right) \leqq-L_{1}<0$ at every points in $\mathscr{U}_{\tilde{k}}$. By $(13), \boldsymbol{K}\left(\mu^{*} \omega_{\mathbf{W}}\right) \leqq-\frac{1}{2}$ in $\mathbf{M} \backslash \boldsymbol{\Delta} \cup \boldsymbol{\Delta}^{*}$. From Lemma 2 , in $\mathscr{U}_{\tilde{k}} \backslash\left(\boldsymbol{\Delta} \cup \boldsymbol{\Delta}^{*}\right)$,

$$
\boldsymbol{K}\left(\Phi_{c, \tilde{k}}\right)=\boldsymbol{K}\left(\mu^{*} \omega_{\mathbf{W}}+c \tilde{\boldsymbol{\omega}}_{\tilde{k}}\right) \leqq-L_{1} /\left(c+2 L_{1}\right)<0 .
$$

By a continuity argument, $\Phi_{c, \tilde{k}}$ has neg. hol. sect. curvature in $\mathscr{U}_{\tilde{k}}$ for any fixed $c$.

Inside the compact set $\mathbf{M} \backslash \mathscr{U}_{\tilde{k}}, \lim _{c \rightarrow 0} \Phi_{c, \tilde{k}}=\mu^{*} \omega_{\mathbf{w}}$ which has neg. hol. sect. curvature. So if $c$ is chosen small enough, $\Phi_{c, \tilde{k}}$ will have neg. hol. sect. curvature in the whole manifold $\mathbf{M}$. This completes the proof of Theorem 2. 


\section{References}

1. Barth, W., Peters, C., Van de Ven, A.: Compact Complex Surfaces. Ergebnisse der Mathematik und ihrer Grenzgebiete, 3. Folge, Band 4. Berlin Heidelberg New York: Springer 1984

2. Brody, R.: Compact manifolds and hyperbolicity. Trans. Am. Math. Soc. 235, 213-219 (1978)

3. Cheung, C.K.: Negative holomorphic sectional curvature and hyperbolic manifolds. Ph.D. Thesis, UC. Berkeley 1988

4. Cowen, M.J.: Families of negatively curved hermitian manifolds. Proc. Am. Math. Soc. 39, (2) 362-366 (1973)

5. Deschamps-Martin, M.: Propriétés de descente des variétés à fibré cotangent ample. Ann. Inst. Fourier (Grenoble) 33, 39-64 (1985)

6. Grauert, H., Reckziegel, H.: Hermitesche Metriken und normale Familien holomorpher Abbildungen. Math. Z. 89, 108-125 (1965)

7. Jost, J., Yau, S.T.: A strong rigidity theorem for a certain class of compact complex analytic surfaces. Math. Ann. 271, 143-152 (1985)

8. Kobayashi, S.: Hyperbolic Manifolds and Holomorphic Mappings. Pure and Appl. Math., 2. New York: Dekker 1970

9. Kodaira, K.: A certain type of irregular algebraic surfaces. J. Anal. Math. 19, 207-215 (1967)

10. Kodaira, K., Spencer, D.C.: On deformations of complex analytic structures, III, stability theorems for complex structures. Ann. Math. 71, 43-76 (1960)

11. Koiso, N.: Einstein metrics and complex structures. Invent. Math. 73, 71-106 (1983)

12. Mok, N.: The holomorphic or anti-holomorphic character of harmonic maps into irreducible compact quotients of polydiscs. Math. Ann. 272, 197-216 (1985)

13. Schneider, M.: Complex surfaces with negative tangent bundle. Complex Analysis and Algebraic Geometry (Göttingen 1985). Lecture Notes Math. 1194, 150-157. Berlin Heidelberg New York. Springer 1986

14. Siu, Y.T.: The Complex-analyticity of harmonic maps and the strong rigidity of compact Kähler manifolds. Ann. Math. 112, 73-112 (1980)

15. Wu, H.: Normal families of holomorphic mappings. Acta Math. 119, 193-233 (1967)

16. Wu, H.: A remark on holomorphic sectional curvature. Indiana Univ. Math. J. 22, 1103-1108 (1972-1973)

17. Yang, P.: Kähler metrics on fibered manifolds. Proc. Am. Math. Soc. 63, 131-133 (1977)

Received November 3, 1987; in final form December 20, 1987 